\title{
Extended Higgs sectors in the context of supersymmetry at the LHC
}

\author{
Priyotosh Bandyopadhyay ${ }^{1, \star}$ \\ ${ }^{1}$ Dipartimento di Matematica e Fisica "Ennio De Giorgi", \\ Università del Salento and INFN-Lecce, Via Arnesano, 73100 Lecce, Italy \\ and \\ Indian Institute of Technology Hyderabad, Kandi, Sangareddy-502287, Telengana, India
}

\begin{abstract}
We study an extension of the Higgs sector of the Standard Model (SM) with $S U(2)$ triplet and a SM gauge singlet in the context of supersymmetry. For a $Z_{3}$ symmetric superpotential where the singlet field generates the bilinear Higgs mixing term between the two Higgs doublets dynamically. Models like this, often have a very light pseudoscalar as a pseudo-Nambu Goldstone model of an extra $U(1)$ symmetry. We investigate the possibility of such light hidden Higgs boson with the data of the discovered Higgs boson around $125 \mathrm{GeV}$. Along with the neutral sector, the charged Higgs bosons in the triplet extended case are really interesting. For instance, the triplet charged Higgs bosons give rise to new decay mode to $Z W^{ \pm}$along with an $a_{1} W^{ \pm}$final state, due to the existence of a light pseudoscalar. These new modes can be looked for at the LHC in the search for Higgs bosons in other representations of $S U(2)$.
\end{abstract}

\section{Introduction}

The discovery of the Higgs boson around $125 \mathrm{GeV}$ has certainly proved the existence of at least one scalar taking part in the process of electroweak symmetry breaking (EWSB) [1]. The presence of such a scalar has been experimentally verified in its decay modes into $W W^{*}, Z Z^{*}$ and $\gamma \gamma$ [1] at the Large Hadron Collider (LHC). In the midst of searching for SM decay modes, it is also important to investigate other non standard searches which are often motivated by various theoretical constructs.

In this article we are going to illustrate the theoretical possibilities which remain, at this time, wide open from a phenomenological perspective and which define the possible scenarios for an extended Higgs sector, in the context of supersymmetry. These constructions also address other theoretical issues, with exciting phenomenological implications. The extension of the Higgs sector with a singlet superfield can solve the $\mu$ problem in the minimal supersymmetric SM (MSSM) dynamically. Similarly one can introduce an $S U(2)$ triplet superfield, but in this case such dynamical solution is not possible, due to the constraints imposed by the $\rho$ parameter on the vacuum expectation value (v.e.v.) of the triplet [2]. Extending the Higgs sector by a singlet/triplet also gives the possibility of a spontaneous violation of the CP symmetry.

We consider a supersymmetric extension of the Higgs sector by a $S U(2) \times U(1)_{Y}$ singlet and a $Y=0$ hypercharge $S U(2)$ triplet. Additional $Z_{3}$ symmetry of the Lagrangian predicts the existence

^e-mail: priyotosh.bandyopadhyay@le.infn.it,bpriyo@iith.ac.in 
of a light pseudoscalar in some limit of the theory. Such possibility leads to additional decay modes of the Higgs boson around $125 \mathrm{GeV}$ into a pair of light pseudoscalars and/or into a light pseudoscalar accompanied by a $Z$ boson, if kinematically allowed.

The charged Higgs sector is extended due to the triplet but they are fermiophobic leading to distinguishing features. A light charged Higgs boson may now decay into a pseudoscalar and one $W^{ \pm}$ boson. This particular decay is allowed for both doublet and triplet-like charged Higgs bosons. However, the $Y=0$ triplet brings in an additional coupling of the charged Higgs boson to the $Z$ and $W^{ \pm}$ at tree-level, which breaks the custodial symmetry. This can generate a totally new decay mode for the charged Higgs boson into $Z W^{ \pm}$, which is not present in the 2-Higgs doublet model (2HDM) or in the MSSM. Here we are going to discuss such possibilities and elaborate on how to explore them at current and future experiments.

In section 2 we are going to briefly illustrate the model. In section 3 we address the possibility of having a light pseudoscalar and its phenomenology. The charged Higgs boson phenomenology has been discussed in section 4 with conclusions in section 5 .

\section{Model}

As explained in [3] the model contains a SU(2) triplet $\hat{T}$ of zero hypercharge $(Y=0)$ together with a SM gauge singlet $\hat{S}$, added to the superfield content of the MSSM,

$$
W_{T N M S S M}=W_{M S S M}+W_{T S} .
$$

The structure of its triplet and singlet extended superpotential can be written as

$$
W_{T S}=\lambda_{T} \hat{H}_{d} \cdot \hat{T} \hat{H}_{u}+\lambda_{S} \hat{S} \hat{H}_{d} \cdot \hat{H}_{u}+\frac{\kappa}{3} \hat{S}^{3}+\lambda_{T S} \hat{S} \operatorname{Tr}\left[\hat{T}^{2}\right] .
$$

The triplet and doublet superfields are given by

$$
\hat{T}=\left(\begin{array}{cc}
\sqrt{\frac{1}{2}} \hat{T}^{0} & \hat{T}_{2}^{+} \\
\hat{T}_{1}^{-} & -\sqrt{\frac{1}{2}} \hat{T}^{0}
\end{array}\right), \quad \hat{H}_{u}=\left(\begin{array}{c}
\hat{H}_{u}^{+} \\
\hat{H}_{u}^{0}
\end{array}\right), \quad \hat{H}_{d}=\left(\begin{array}{c}
\hat{H}_{d}^{0} \\
\hat{H}_{d}^{-}
\end{array}\right) .
$$

Here $\hat{T}^{0}$ denotes a complex neutral superfield, while $\hat{T}_{1}^{-}$and $\hat{T}_{2}^{+}$are the charged Higgs superfields. The MSSM Higgs doublets are the only superfields which couple to the fermion multiplet via Yukawa coupling. All the coefficients involved in the Higgs sector are chosen to be real in order to preserve $\mathrm{CP}$ invariance. The breaking of the $S U(2)_{L} \times U(1)_{Y}$ electroweak symmetry is then obtained by giving real vevs to the neutral components of the Higgs field

$$
<H_{u}^{0}>=\frac{v_{u}}{\sqrt{2}}, \quad<H_{d}^{0}>=\frac{v_{d}}{\sqrt{2}}, \quad<S>=\frac{v_{S}}{\sqrt{2}}, \quad<T^{0}>=\frac{v_{T}}{\sqrt{2}},
$$

which give mass to the $W^{ \pm}$and $Z$ bosons

$$
m_{W}^{2}=\frac{1}{4} g_{L}^{2}\left(v^{2}+4 v_{T}^{2}\right), \quad m_{Z}^{2}=\frac{1}{4}\left(g_{L}^{2}+g_{Y}^{2}\right) v^{2}, \quad v^{2}=\left(v_{u}^{2}+v_{d}^{2}\right), \quad \tan \beta=\frac{v_{u}}{v_{d}}
$$

and also induce, as mentioned above, a $\mu$-term of the form $\mu_{D}=\frac{\lambda_{S}}{\sqrt{2}} v_{S}+\frac{\lambda_{T}}{2} v_{T}$. The triplet vev $v_{T}$ is strongly constrained by the global fit to the measured value of the $\rho$ parameter [2] which restricts its value to $v_{T} \leq 5 \mathrm{GeV}$ and in the numerical analysis we have chosen $v_{T}=3 \mathrm{GeV}$. 
In the TNMSSM, we are left with four CP-even, three CP-odd and three charged Higgs bosons as shown below

$$
\begin{array}{ccc}
\mathrm{CP}-\text { even } & \mathrm{CP}-\text { odd } & \text { charged } \\
h_{1}, h_{2}, h_{3}, h_{4} & a_{1}, a_{2}, a_{3} & h_{1}^{ \pm}, h_{2}^{ \pm}, h_{3}^{ \pm} .
\end{array}
$$

The neutral Higgs bosons are linear combinations of doublets, triplet and singlets, whereas the charged Higgses are combinations of doublets and of a triplet only. At tree-level the maximum value of the lightest neutral Higgs has additional contributions from the triplet and the singlet sectors respectively. The upper bound on the lightest CP-even Higgs can be extracted from the relation

$$
m_{h_{1}}^{2} \leq m_{Z}^{2}\left(\cos ^{2} 2 \beta+\frac{\lambda_{T}^{2}}{g_{L}^{2}+g_{Y}^{2}} \sin ^{2} 2 \beta+\frac{2 \lambda_{S}^{2}}{g_{L}^{2}+g_{Y}^{2}} \sin ^{2} 2 \beta\right),
$$

which is affected on its right-hand-side by two additional contributions from the triplet and the singlet. These can raise the allowed tree-level Higgs mass and additional contributions coming from the radiative corrections of the triplet and the singlet reduce the required the supersymmetric (SUSY) mass scale and thus the fine-tuning problem. We have calcualted the one-loop Higgs mass for the neutral Higgs bosons in [3] following the Coleman-Weinberg effective potential [4]. The existence of the triplet and singlet fields at quantum level are non-negligible which reduce the SUSY fine-tuning for all $\tan \beta$ values $[3,5,6]$.

\section{Light pseudoscalar and phenomenology}

One of the most interesting feature can be seen in the limit where the trilinear soft parameters $A_{i}$ in go to zero, the discrete $Z_{3}$ symmetry of the Lagrangian is promoted to a continuos $U(1)$ symmetry given by Eq. (7)

$$
\left(\hat{H}_{u}, \hat{H}_{d}, \hat{T}, \hat{S}\right) \rightarrow e^{i \phi}\left(\hat{H}_{u}, \hat{H}_{d}, \hat{T}, \hat{S}\right)
$$

An explicit breaking of a continuous global symmetry is expected to be accompanied by pseudoNambu Goldstone bosons (pNGB) as in the case of chiral symmetry breaking in QCD where the pions take the role of the corresponding pNGB. In this case, in the TNMSSM we should expect a light pseudoscalar in the spectrum, whose mass is of the same order of the $A_{i}$ parameters. A similar behaviour is allowed in the NMSSM and such light pseudoscalar is known as the $R$-axion [7,8]. However, such light pseudoscalar receives bounds if it decays into fermions. Previous analysis at LEP have searched for such light scalar bosons in $e^{+} e^{-} \rightarrow Z h$ and $e^{+} e^{-} \rightarrow A h$, where $h, A$ are CP-even and odd neutral Higgs bosons respectively [9]. Similar bounds also come from the bottomonium decay [10] for such light pseudoscalar in the mass range of 5.5 -14 GeV. Recently published data from CMS also provide strong bounds for such a light pseudoscalar when it couples to fermions [11]. Being mostly singlet-like, it is easy to evade such bounds but difficult to produce it directly at a hadron collider.

In Figure 1 we show a Higgs $\sim 125 \mathrm{GeV}$ which at the same time satisfy the $Z Z^{*}, W W^{*}$ bounds at $1 \sigma$ level and the $\gamma \gamma$ bound at $2 \sigma$ level from both CMS and ATLAS along with a light pseudoscalar with or without a light scalar. Such singlet type light pseudoscalar couples to the doublet-like Higgs bosons via $\lambda_{S}$ (see Eq. 2). This makes it easy to produce by an intermediate Higgs. If such a light pseudoscalar $\left(m_{a_{1}} \leq 125 / 2\right)$ exists, then it can be produced in $g g \rightarrow h_{125} \rightarrow a_{1} a_{1}$ as shown in Figure 2. Such pair production can give rise to final states rich in $\tau, b$ and even in muons. In [12] 


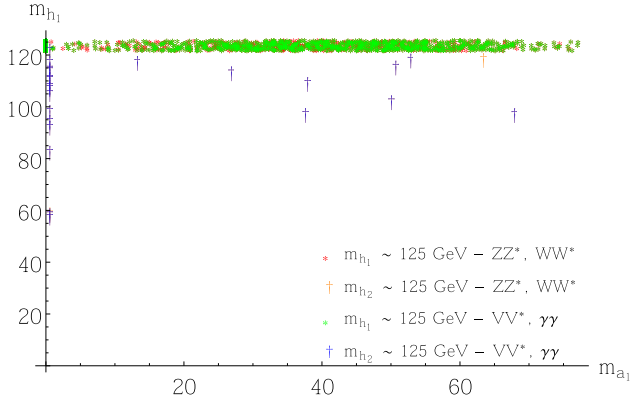

(a)

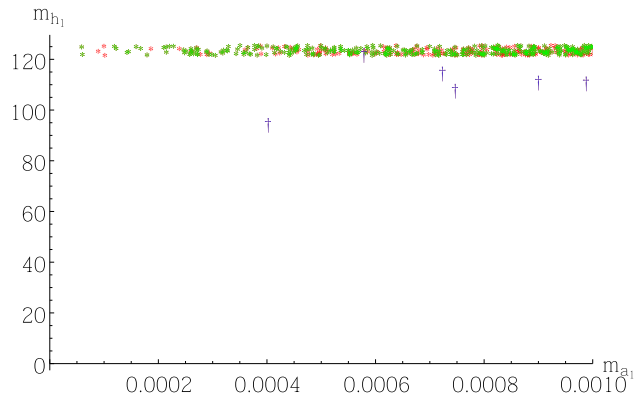

(b)

Figure 1. The lightest CP-even Higgs boson mass $m_{h_{1}}$ vs the lightest pseudoscalar mass $m_{a_{1}}$ at one-loop consistent with the Higgs data from CMS, ATLAS and LEP. Very light pseudoscalar masses $m_{a_{1}} \leq 1 \mathrm{MeV}$ are shown in panel (b)[3].

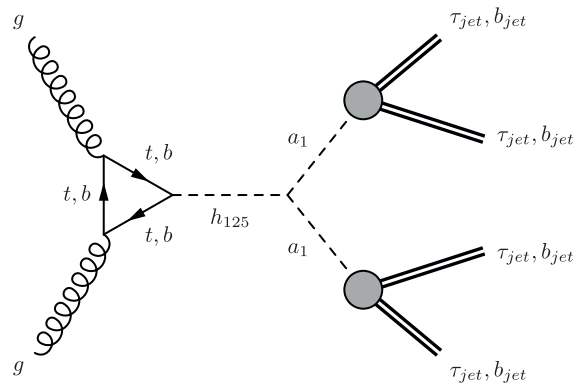

Figure 2. Pseudoscalar (triplet/singlet) pair production from Higgs boson, generated via gluon-gluon fusion, and their decays via their mixing with the doublets [12].

we have investigated such final states by considering all the dominant SM backgrounds at the LHC with a center of mass energy of 13 and $14 \mathrm{TeV}$. A detailed signal to background analysis of the final states $2 \tau+2 b, 2 b+2 \mu$ and $\geq 3 \tau$ reveals that some of the benchmark points with the light pseudoscalar $\left(m_{a_{1}} \sim 10-20 \mathrm{GeV}\right)$ can be probed with early data of $25 \mathrm{fb}^{-1}$ at the LHC.

\section{Charged Higgs boson phenomenology}

As mentioned earlier that unlike in the NMSSM and MSSM, the TNMSSM has three charged Higgs bosons out of which two of them are triplets. Henceforth, they do not couple to fermions and are hard to produce directly. In the mass basis they are generally in mixed states. The presence of a light pseudoscalar affects the charged Higgs decay phenomenology and such light state can be probed via $h_{1}^{ \pm} \rightarrow a_{1} W^{ \pm}$. Similarly in the case of the NMSSM with a $Z_{3}$ symmetry [7], the non-standard decay mode of the light charged Higgs boson $h^{ \pm} \rightarrow a W^{ \pm}$can evade the recent mass bounds from the LHC [13] and can be probed with the $\tau$ and $b$ rich final states coming from the light pseudoscalar [14]. The charged Higgs boson in this case (NMSSM) is still doublet-like so its production from the decay of a 


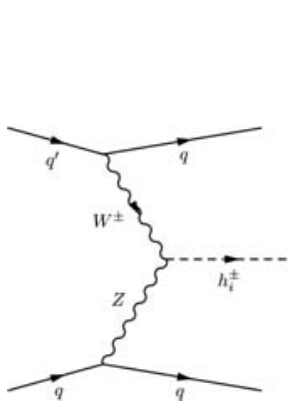

(a)

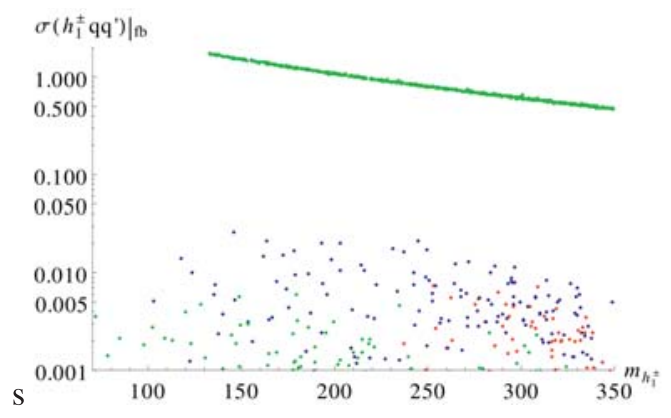

(b)

Figure 3. The Feynman diagram for the charged Higgs production via vector boson fusion at the LHC (a) and the production cross-section of a light charged Higgs boson via vector boson fusion versus the light charged Higgs boson mass $m_{h_{1}^{ \pm}}(\mathrm{b})[16]$.

top or via $b g \rightarrow t h^{ \pm}$is still possible. The possibility of a light scalar is also present in the MSSM with CP-violating interactions, where the light Higgs is mostly CP-odd and can evade the existing LEP bounds. Such Higgs boson with a mass $\lesssim 30 \mathrm{GeV}$ can open the decay mode $h^{ \pm} \rightarrow h_{1} W^{ \pm}$, which can be explored at the LHC [15].

However things can change a lot in a triplet extended supersymmetric model (TESSM), where we have a light charged Higgs triplet. It does not couple to fermions, which makes its production via conventional methods a lot harder. However, its production channels in association with $W^{ \pm}$bosons or with neutral Higgs bosons are still considerable. The interesting feature of having a charged Higgs triplet is the non-zero $h_{i}^{ \pm}-Z-W^{\mp}$ coupling, which not only gives rise to a new decay mode of $Z W^{ \pm}$but also makes the charged Higgs production possible via vector boson fusion [16] as can be seen from Fig 3. This is not allowed for the doublet-like charged Higgs boson of the MSSM or of the 2HDM. In the TNMSSM we have both the presence of a very light pseudoscalar - as in the NMSSM - and of a charged Higgs triplet, as in the TESSM. If we can pair produce such charged Higgs where one of them decays into $a_{1} W^{ \pm}$and the other into $Z W^{ \pm}$, then we can search for such decay modes. This provides a smoking gun signature for the extended Higgs structure of the TNMSSM. We are currently investigating in detail ways to probe both aspects at the LHC [17].

\section{Conclusions}

The discovery of a Higgs boson around $125 \mathrm{GeV}$ in mass cannot explain all the shortcomings of the SM, and the quest for extra Higgs bosons are still there. Supersymmetric theories allow additional Higgs bosons which may belong to the usual or to higher representations of $S U(2)$. Phenomenologically, the existence of a very light scalar below $100 \mathrm{GeV}$ in mass is still open, together with the theoretical possibility of various representations. Given that all the SM decay modes of the $125 \mathrm{GeV}$ Higgs boson are yet to be discovered, the question of the existence of non-standard decays in this sector still remains elusive. The other Higgs bosons could have higher mass values $(>125 \mathrm{GeV})$ but the possibilities of lower mass values $(<100 \mathrm{GeV})$ also remain quite open. Certainly in such cases, if the $h_{125} \rightarrow h_{1} h_{1} / a_{1} a_{1}$ decay modes are kinematically allowed, then they can be probed at the LHC. Models with $Z_{3}$ symmetry, such as the NMSSM or the TNMSSM, where such scalars are naturally light, can be tested once additional data will be made available from the LHC. If an extended Higgs 
sector exists, then finding a charged Higgs is a direct proof of it. So far, the search for a charged Higgs boson is performed in its decay to $\tau v$, but the existence of a light scalar/pseudoscalar gives us the new decay mode $h^{ \pm} \rightarrow a_{1} / h_{1} W^{ \pm}$, which should be looked for at the LHC. Triplet charged and neutral Higgs bosons do not couple to fermions, and a charged Higgs triplets can decay to $Z W^{ \pm}$which can be explored at the LHC. To complete our understanding of electroweak phase transition we need to explore all the theoretical possibilities experimentally.

\section{Acknowledgement}

PB wants to thank University of Salento for the invitation and support to attend the workshop.

\section{References}

[1] G. Aad et al. [ATLAS and CMS Collaborations], arXiv:1503.07589 [hep-ex].

[2] J. Beringer et al. [Particle Data Group Collaboration], Phys. Rev. D 86 (2012) 010001.

[3] P. Bandyopadhyay, C. Corianò and A. Costantini, JHEP 1509 (2015) 045 [arXiv:1506.03634 [hep-ph]].

[4] S. R. Coleman and E. J. Weinberg, Phys. Rev. D 7 (1973) 1888.

[5] P. Bandyopadhyay, K. Huitu and A. Sabanci, JHEP 1310 (2013) 091 [arXiv:1306.4530 [hep-ph]].

[6] P. Bandyopadhyay, S. Di Chiara, K. Huitu and A. S. Keceli, JHEP 1411 (2014) 062 [arXiv:1407.4836 [hep-ph]]. S. Di Chiara and K. Hsieh, Phys. Rev. D 78 (2008) 055016 [arXiv:0805.2623 [hep-ph]].

[7] U. Ellwanger, C. Hugonie and A. M. Teixeira, Phys. Rept. 496 (2010) 1 [arXiv:0910.1785 [hep$\mathrm{ph}]]$.

[8] K. Agashe, A. Azatov, A. Katz and D. Kim, Phys. Rev. D 84 (2011) 115024 [arXiv:1109.2842 [hep-ph]].

[9] R. Barate et al. [LEP Working Group for Higgs boson searches and ALEPH and DELPHI and L3 and OPAL Collaborations], Phys. Lett. B 565 (2003) 61 [hep-ex/0306033]. S. Schael et al. [ALEPH and DELPHI and L3 and OPAL and LEP Working Group for Higgs Boson Searches Collaborations], Eur. Phys. J. C 47 (2006) 547 [hep-ex/0602042].

[10] P. Franzini, D. Son, P. M. Tuts, S. Youssef, T. Zhao, J. Lee-Franzini, J. Horstkotte and C. Klopfenstein et al., Phys. Rev. D 35 (1987) 2883.

[11] S. Chatrchyan et al. [CMS Collaboration], Phys. Rev. Lett. 109 (2012) 121801 [arXiv:1206.6326 [hep-ex]].

[12] P. Bandyopadhyay, C. Corianò and A. Costantini, JHEP 1512 (2015) 127 [arXiv:1510.06309 [hep-ph]].

[13] CMS Collaboration [CMS Collaboration], CMS-PAS-HIG-14-020. G. Aad et al. [ATLAS Collaboration], JHEP 1503 (2015) 088 [arXiv:1412.6663 [hep-ex]].

[14] P. Bandyopadhyay, K. Huitu and S. Niyogi, JHEP 1607 (2016) 015 [arXiv:1512.09241 [hep$\mathrm{ph}]]$.

[15] P. Bandyopadhyay, JHEP 1108 (2011) 016 [arXiv:1008.3339 [hep-ph]].

P. Bandyopadhyay and K. Huitu, JHEP 1311 (2013) 058 [arXiv:1106.5108 [hep-ph]]. P. Bandyopadhyay, A. Datta, A. Datta and B. Mukhopadhyaya, Phys. Rev. D 78 (2008) 015017 [arXiv:0710.3016 [hep-ph]].

[16] P. Bandyopadhyay, C. Corianò and A. Costantini, arXiv:1512.08651 [hep-ph]. P. Bandyopadhyay, K. Huitu and A. S. Keceli, JHEP 1505 (2015) 026 [arXiv:1412.7359 [hep-ph]].

[17] P. Bandyopadhyay, and A. Costantini, in preparation. 